\title{
Comparison of Therapeutic Effects of Intra and Extra Oral Low Level Laser Radiation in Maxillary Chronic Sinusitis
}

\author{
Hamed Mortazavi ${ }^{1}$, Robab Noormohammadi ${ }^{2, *}$, Hamidreza Khalighi ${ }^{1}$, Ali Goljanian ${ }^{3}$, \\ Masoud Mojahedi ${ }^{4}$, Siamak Sabour ${ }^{5}$ \\ ${ }^{1}$ Department of Oral Medicine, Dental School, Shahid Beheshti University of Medical Sciences, Tehran, Iran \\ ${ }^{2}$ Department of Oral Medicine, Dental School, Zanjan University of Medical Sciences, Zanjan, Iran \\ ${ }^{3}$ Department of Otolaryngology, Medical School, Shahid Beheshti University of Medical Sciences, Tehran, Iran \\ ${ }^{4}$ Department of Laser, Dental School, Shahid Beheshti, University of Medical Sciences, Tehran, Iran \\ ${ }^{5}$ Department of Clinical Epidemiology, Dental School, Shahid Beheshti University of Medical Sciences, Tehran, Iran \\ Email address: \\ hamedmortazavi2013@gmail.com (H. Mortazavi),robab.noormohammadi@yahoo.com(R. Noormohammadi), \\ dr.hrkh92@gmail.com (H. Khalighi), ali.goljanian@gmail.com (A. Goljanian), seyed220@gmail.com (M. Mojahedi), \\ s.sabour@gmail.com (S. Sabour) \\ ${ }^{*}$ Corresponding author
}

\section{To cite this article:}

Hamed Mortazavi, Robab Noormohammadi, Hamidreza Khalighi, Ali Goljanian, Masoud Mojahedi, Siamak Sabour. Comparison of Therapeutic Effects of Intra and Extra Oral Low Level Laser Radiation in Maxillary Chronic Sinusitis. International Journal of Dental Medicine. Vol. 5, No. 1, 2019, pp. 19-28. doi: 10.11648/j.ijdm.20190501.14

Received: March 2, 2019; Accepted: April 10, 2019; Published: May 6, 2019

\begin{abstract}
Chronic sinusitis is one of the most common chronic diseases. In this research, intra- oral radiation of low-level laser has been compared with extra-oral low level laser radiation in treating maxillary chronic sinusitis for the first time. In this clinical trial, 40 patients were enrolled. Treatment plan was performed in 8 sessions, every other day, and using low-level diode laser with $810 \mathrm{~nm}$. SNOT-22 questionnaire and rhinomanometry were used for evaluating patients. Mann whiteny, Fridman and wilcoxson tests were used for data analyses. P. value $<0.05$ was considered statistically significant. All studied variables were improved using intra and extra oral low-level laser and this improvement was statistically significant (P. value $<0.05)$. Comparing the two groups, Intra-oral laser therapy significantly $(\mathrm{P}$. value $<0.05)$ improved rhinomanometry variables compared to extra-oral laser therapy. The two groups showed no significant difference in improvement of clinical symptoms that contained in the SNOT-22 questionnaire except for sneeze which intra-oral laser therapy significantly decreased this variable (P. value $<0.05$ ). It should be noted that intra-oral radiation reduced all symptoms earlier than extra-oral radiation. After Sixmonths, in intra-oral group there was no significant difference between the results of completion and the results of 8th treatment session (P. value $>0.05$ ). But in extra-oral group only post nasal discharge and thick nasal discharge were significantly increased compared with the results of the 8th session of radiation. Other symptoms showed no significant difference (P. value>0.05). Using intra and extra oral low-level laser therapy is a suitable way to treat patients with chronic maxillary sinusitis.
\end{abstract}

Keywords: Chronic Sinusitis, Maxillary Sinusitis, Low-Level Laser Therapy, Extra Oral and Intra Oral Laser Therapy

\section{Introduction}

Sinusitis is one of the most common diagnoses in primary care. [1] It is one of the most common chronic diseases, affecting $14-16 \%$ of the adult US population and highly prevalent and has a considerable impact on quality of life and health care expenditures. [2, 3] Because the nature and etiology of chronic sinusitis are not completely unknown, there is not any standard treatment for this disease [4] Kinds of treatment of CRS is aimed at reducing underlying inflammation and facilitating clearance of the paranasal sinuses. Antibiotics, topical steroids, systemic steroids, and nasal saline irrigation are mainstays of treatment. [1] Chronic nature of CRS has influenced the treatment why needs long 
term treatment and long-term use of systemic drugs certainly has many side effects. For example, in the short term, antibiotics reduce bacterial diversity even more and allow colonization with microbiota that is less susceptible to the prescribed antibiotics. Many bacteria residing on mucosal surfaces exist in a biofilm state, making them resistant to most antibiotics. The biofilm and the bacteria in mature biofilms being metabolically silent, and thus not taking up and/or metabolizing antibiotics [5] AS a result, Researchers have suggested local treatment [6]. But there is inadequate data to promote the use of oral steroids in CRS without nasal polyps. And topical antibiotics do not show benefit in CRS without nasal polyps [6]. In the patients without anatomical obstruction, functional endoscopic surgeries are not superior to drug therapies. Therefore, because of these difficulties in treating chronic sinusitis, researchers and clinicians suggested new modalities such as ultrasound therapy and laser therapy [6] Therapeutic ultrasound and laser therapy are blocking molecular communication between bacteria, inhibiting biofilm production and disrupting bacterial biofilms [6-8] low level laser therapy LLLT is a form of phototherapy that delivers low-power $(\leq 500 \mathrm{mw})$ coherent and collimated beam of light of a single wavelength [6]. LLLT is attributed to non-thermal events. It involves the absorption of photon radiation by chromophores such as cytochrome $\mathrm{C}$ oxidase within the mitochondria leading to release of nitric oxide (NO), and an increase in ATP levels. These events can lead to modulation of cell metabolism, normalization of cell function, inflammation reduction, pain relief, and tissue repair $[6,9]$. There is strong evidence that LLLT has anti-inflammatory effects [6]. In a recent in vitro study, researchers demonstrated that laser has bacterial biofilm treatment potential; a significant bacterial count reduction was achieved in an animal model of acute bacterial rhinosinusitis [10]. In evaluating therapeutic outcomes of $\mathrm{CRS}$, it is better to consider subjective and objective factors at the same time [11]. Although CT provided detailed information on sinus involvement; but its relation with symptom severity is not reliable [12] Bedside it's kind of an expensive imaging method [12] And also according to this fact that nasal obstruction is the most common (81-95\%) clinical symptoms in CRS [13, 14], researchers of this study began using rhinomanometry which helps them evaluate clinical symptoms objectively and with lower expenditure. There are few studies on therapeutic effects of low-level laser therapy in treating chronic sinusitis $[6-8,10]$. However, this study is the first long-term clinical study to compare therapeutic effects of intra and extra oral low-level laser radiation in maxillary chronic sinusitis.

\section{Material and Experiments}

This study is experimental (interventional) study and performed in before/after manner on 40 patients with chronic sinusitis in Dental school, Shaheed Beheshti University of medical sciences, Tehran, Iran, between 2012-2014. Objectives of the study were explained to patients and written consent was obtained from each of them before performing it. This study was registered in ClinicalTrials.gov under NCT02124538. Physical examination of all patients was performed by otolaryngologist in Taleqani hospital. Patients' information was recorded based upon information forms. Symptom improvement was evaluated in two sections: 1- subjective; which was based on patients' answers in questionnaires. SNOT-22 questionnaire was used in this study, which was validated in 2009 , and is recommended in clinical evaluations $[15,16]$. There are 22 questions in this questionnaire including: Need to blow nose, Sneezing, Runny nose, Cough, Post nasal discharge, Thick nasal discharge, Ear fullness, Dizziness, Ear pain, Facial pain/pressure, Difficulty falling asleep, Waking up at night, Lack of a good night's sleep, Waking up tired, Fatigue, Low performance, Reduced concentration, Frustrated/restless, Sadness, Embarrassment, Sense of taste/smell, Blockage/congestion of nose. All these symptoms were assessed in six grades ranging from having no problem to having severe problems. 2- Objective; which was performed by otolaryngologist using rhinomanoetry test (Rhinomanometry: Ecleris, Rhinosoft, Argentina). In this test, resistance and air flow were measured simultaneously in nasal cavity. Patients should have these criteria: 1- affected by chronic sinusitis: a chronic inflammatory process affecting paranasal sinuses and nasal mucosa, lasting at least 12 weeks and the patient must have two major clinical symptoms or one major and two minor clinical symptoms. 2- Being healthy 3- Not being pregnant or in breast feeding state. 4Should not have sinus surgery, nasal septum deviation, and nasal polyp. 5- Being cooperative in research. If the patient did not have any of these inclusion criteria, he/she would have been excluded from the study. After confirming the disease and recording rhinomanometry curve in the forms and also filling SNOT22 questionnaire for the first time, the patient had been irradiated with low-level laser (Dr. Smile, low level, LAMBDA SpA 5 (Company), France). Intra-oral laser radiation in vestibule depth from canine apical zone to first molar apical zone (floor of the maxillary sinus was performed with 45 degree and without pressure in 10 points with $3 \mathrm{~mm}$ distances). This was performed by one person under supervision of laser specialist; then bias probability was decreased. In this method, laser radiation with $810 \mathrm{~nm}$ and $0.1,0.4,0.3,0.2 \mathrm{w}$ by single probe is irradiated from buccal side. Extra-oral radiation was performed along the ala-tragus line with an approximately fifteen to thirty negative degrees to the zygomatic arch (depending on the anatomy of zygomatic arch). Ten points with three-millimeter intervals, were irradiated with pressure for 60 seconds and after 60 seconds of rest, laser flashed at the next point with the same condition. Intraoral radiation was done without pressure.

Irradiation was performed every other day with the highest power $-0.4 \mathrm{~W}$ - and ended in lowest power $0.1 \mathrm{~W}$ and this cycle repeated two times. (The first day, $0.4 \mathrm{~W}$; second day, rest; third day, $0.3 \mathrm{~W}$; forth day, rest; fifth day, $0.2 \mathrm{~W}$; sixth day, rest; and finally seventh day, $0.1 \mathrm{~W}$ ). Every session 
lasted 40 minutes including resting time (activity time of machine was 20 minutes). Depending on different powers in different days, total dose of energy was 4-7 Joules in every session. There were 8 sessions of irradiation [14]. At the end of every session, questionnaires were filled again by patients. In the last session, otolaryngologist again assessed the patients with physical examination and rhinomanometry. Then, the data collected by these questionnaires and also the results of rhinomanometry had been statistically analyzed. Six months after the last treatment, we called patients and the questionnaires were filled again based on their opinion. The results of these calls were also analyzed.

\subsection{Ethical Consideration}

This study was approved by the ethics committee of Shaheed Beheshti University of Medical Science and written informed consent was obtained from all patients enrolled in the study.

\subsection{Data Analysis}

Data were analyzed using SPSS software version 18.0. Quantitative and qualitative variables were described by means and standard deviation and number and percentage. We used Friedman and Wilcoxon and Non-parametric Mann-
Whitney tests for data analysis. $\mathrm{P}$ value $<0.05$ was determined significant.

\section{Results}

This study included 40 patients with chronic maxillary sinusitis. 20 patients (12 women and 8 men) were in intraoral group and 20 patients ( 9 women and 11 men) participated in extra-oral laser radiation. 4 patients were excluded because of dissociation. Average age of intra-oral \&

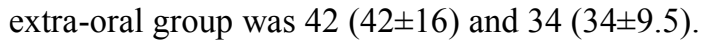

\subsection{The Results of SNOT22 Questionnaire}

The results obtained about 22 clinical symptoms in this questionnaire are summarized in table 1. (Due to length of eight sessions of radiation results, only the results of sessions before of radiation, the fourth and the eighth session are shown in Table 1. In analysis level, we used Friedman test for evaluating whether there is significant decrease of SNOT-22 variables or not. The results show that intra-oral and extraoral radiation decreased these variables significantly (P. value $=0.001)$ Then, Non-parametric Mann-Whitney test was used to compare two groups.

Table 1. Results from the analysis of variables in snot-22 questionnaire (In Sessions before/middle/end of intra and extra irradiation).

\begin{tabular}{|c|c|c|c|c|c|c|}
\hline & \multicolumn{3}{|l|}{ Intra-oral } & \multicolumn{3}{|l|}{ Extra-oral } \\
\hline & $\begin{array}{l}\text { Session Before } \\
\text { irradiation }\end{array}$ & Fourth session & Eighth Session & $\begin{array}{l}\text { Session Before } \\
\text { irradiation }\end{array}$ & Fourth session & Eighth Session \\
\hline & \multicolumn{6}{|l|}{ need to blow nose } \\
\hline no problem & 18.2 & 33.3 & 39.4 & 15.2 & 27.3 & 36.4 \\
\hline very mild problem & 6.1 & 6.1 & 6.1 & 12.1 & 6.1 & 9.1 \\
\hline mild problem & 9.1 & 9.1 & 6.1 & 9.1 & 12.1 & 3 \\
\hline moderate problem & 12.1 & 3 & 0 & 9.1 & 3 & 0 \\
\hline problem as bad as it can be & 0 & 0 & 0 & 0 & 0 & 0 \\
\hline P. value (Wilcoxon) & - & 0.003 & 0.003 & - & 0.024 & 0.007 \\
\hline P. value (Friedman) & 0.001 & & & 0.001 & & \\
\hline P. value Mann. Whiteny (before) & 0.61 & & & & & \\
\hline \multirow[t]{2}{*}{ P. value Mann. Whiteny (after) } & 1 & & & & & \\
\hline & Runny nose & & & & & \\
\hline no problem & 12.1 & 21.2 & 36.4 & 9.1 & 9.1 & 24.2 \\
\hline mild problem & 12.1 & 15.2 & 9.1 & 9.1 & 6.1 & 0 \\
\hline moderate problem & 9.1 & 0 & 0 & 12.1 & 6.1 & 3 \\
\hline severe problem & 6.1 & 3 & 0 & 3 & 0 & 0 \\
\hline problem as bad as it can be & 6.1 & 0 & 0 & 3 & 0 & 0 \\
\hline P. value (Wilcoxon) & - & 0.003 & 0.003 & - & 0.006 & 0.007 \\
\hline P. value (Friedman) & 0.001 & & & 0.001 & & \\
\hline P. value Mann. Whiteny (before) & 0.81 & & & & & \\
\hline \multirow[t]{2}{*}{ P. value Mann. Whiteny (after) } & 0.4 & & & & & \\
\hline & post nasal discharg & & & & & \\
\hline no problem & 0 & 3 & 27.3 & 0 & 0 & 24.2 \\
\hline very mild problem & 0 & 6.1 & 24.2 & 0 & 0 & 15.2 \\
\hline mild problem & 0 & 12.1 & 0 & 0 & 15.2 & 6.1 \\
\hline moderate problem & 9.1 & 21.2 & 0 & 18.2 & 12.1 & 3 \\
\hline severe problem & 27.3 & 6 & 0 & 24.2 & 21.2 & 0 \\
\hline problem as bad as it can be & 15.2 & 3 & 0 & 6.1 & 0 & 0 \\
\hline P. value (Wilcoxon) & - & 0.001 & 1 & - & 0.001 & 0.001 \\
\hline P. value Mann. Whiteny (after) & 0.52 & & & & & \\
\hline
\end{tabular}




\begin{tabular}{|c|c|c|c|c|c|c|}
\hline & \multicolumn{3}{|l|}{ Intra-oral } & \multicolumn{3}{|l|}{ Extra-oral } \\
\hline & $\begin{array}{l}\text { Session Before } \\
\text { irradiation }\end{array}$ & Fourth session & Eighth Session & $\begin{array}{l}\text { Session Before } \\
\text { irradiation }\end{array}$ & Fourth session & Eighth Session \\
\hline & ear fullness & & & & & \\
\hline no problem & 24.2 & 27.3 & 39.4 & 15.2 & 18.2 & 30.3 \\
\hline very mild problem & 9.1 & 12.1 & 6.1 & 3 & 12.1 & 6.1 \\
\hline mild problem & 3 & 6.1 & 6.1 & 9.1 & 9.1 & 9.1 \\
\hline moderate problem & 6.1 & 3 & 0 & 9.1 & 9.1 & 3 \\
\hline severe problem & 6.1 & 3 & 0 & 12.1 & 0 & 0 \\
\hline problem as bad as it can be & 3 & 0 & 0 & 0 & 0 & 0 \\
\hline P. value (Wilcoxon) & - & 0.039 & 0.026 & - & 0.006 & 0.007 \\
\hline P. value (Friedman) & 0.001 & & & 0.001 & & \\
\hline P. value Mann. Whiteny (before) & 0.31 & & & & & \\
\hline P. value Mann. Whiteny (after) & $\begin{array}{l}0.32 \\
\text { ear pain }\end{array}$ & & & & & \\
\hline no problem & 21.2 & 30.3 & 39.4 & 24.2 & 27.3 & 30.3 \\
\hline very mild problem & 9.1 & 12.1 & 9.1 & 6.1 & 12.1 & 12.1 \\
\hline mild problem & 9.1 & 3 & 3 & 3 & 6.1 & 6.1 \\
\hline moderate problem & 3 & 6.1 & 0 & 15.2 & 3 & 0 \\
\hline severe problem & 6.1 & 0 & 0 & 0 & 0 & 0 \\
\hline problem as bad as it can be & 3 & 0 & 0 & 0 & 0 & 0 \\
\hline P. value (Wilcoxon) & - & 0.016 & 0.016 & - & 0.06 & 0.026 \\
\hline P. value (Friedman) & 0.001 & & & 0.001 & & \\
\hline P. value Mann. Whiteny (before) & 0.78 & & & & & \\
\hline \multirow[t]{2}{*}{ P. value Mann. Whiteny (after) } & 0.37 & & & & & \\
\hline & \multicolumn{3}{|c|}{ difficulty falling asleep } & & & \\
\hline no problem & 3 & 9.1 & 33.3 & 15.2 & 18.2 & 30.3 \\
\hline very mild problem & 12.1 & 24.2 & 15.2 & 15.2 & 18.2 & 12.1 \\
\hline mild problem & 15.2 & 18.2 & 3 & 6.1 & 6.1 & 6.1 \\
\hline moderate problem & 12.1 & 0 & 0 & 9.1 & 6.1 & 0 \\
\hline severe problem & 9.1 & 0 & 0 & 3 & 0 & 0 \\
\hline problem as bad as it can be & 0 & 0 & 0 & 0 & 0 & 0 \\
\hline P. value (Wilcoxon) & - & 0.003 & 0.001 & - & 0.034 & 0.023 \\
\hline P. value (Friedman) & 0.001 & & & 0.001 & & \\
\hline P. value Mann. Whiteny (before) & $0.05^{*}$ & & & & & \\
\hline \multirow[t]{2}{*}{ P. value Mann. Whiteny (after) } & 0.79 & & & & & \\
\hline & \multicolumn{3}{|c|}{ Lack of good night sleep } & & & \\
\hline no problem & 27.3 & 9.1 & 27.3 & 27.3 & 27.3 & 30.3 \\
\hline very mild problem & 3 & 21.2 & 15.2 & 3 & 9.1 & 15.2 \\
\hline mild problem & 0 & 12.1 & 6.1 & 0 & 6.1 & 3 \\
\hline moderate problem & 9.1 & 6.1 & 3 & 9.1 & 6.1 & 0 \\
\hline severe problem & 6.1 & 3 & 0 & 6.1 & 0 & 0 \\
\hline problem as bad as it can be & 3 & 0 & 0 & 3 & 0 & 0 \\
\hline P. value (Wilcoxon) & - & 0.01 & 0.002 & - & 0.041 & 0.024 \\
\hline P. value (Friedman) & 0.001 & & & 0.001 & & \\
\hline P. value Mann. Whiteny (before) & 0.07 & & & & & \\
\hline P. value Mann. Whiteny (after) & $\begin{array}{l}0.45 \\
\text { fatigue }\end{array}$ & & & & & \\
\hline no problem & 9.1 & 12.1 & 27.3 & 6.1 & 9.1 & 27.3 \\
\hline very mild problem & 6.1 & 15.2 & 12.1 & 3 & 12.1 & 12.1 \\
\hline mild problem & 9.1 & 12.1 & 9.1 & 3 & 15.2 & 6.1 \\
\hline moderate problem & 9.1 & 9.1 & 0 & 15.2 & 12.1 & 3 \\
\hline severe problem & 12.1 & 3 & 3 & 18.2 & 0 & 0 \\
\hline problem as bad as it can be & 6.1 & 0 & 0 & 3 & 0 & 0 \\
\hline P. value (Wilcoxon) & - & 0.008 & 0.015 & - & 0.001 & 0.001 \\
\hline P. value (Friedman) & 0.001 & & & 0.001 & & \\
\hline P. value Mann. Whiteny (before) & 0.48 & & & & & \\
\hline \multirow[t]{2}{*}{ P. value Mann. Whiteny (after) } & \multirow{2}{*}{\multicolumn{3}{|c|}{$\begin{array}{l}0.79 \\
\text { reduced concentration }\end{array}$}} & & & \\
\hline & & & & & & \\
\hline no problem & 24.2 & 27.3 & 36.4 & 12.1 & 15.2 & 36.4 \\
\hline very mild problem & 6.1 & 9.1 & 6.1 & 9.1 & 18.2 & 6.1 \\
\hline mild problem & 6.1 & 15.2 & 9.1 & 9.1 & 15.2 & 6.1 \\
\hline moderate problem & 12.1 & 0 & 0 & 6.1 & 0 & 0 \\
\hline severe problem & 3 & 0 & 0 & 9.1 & 0 & 0 \\
\hline problem as bad as it can be & 0 & 0 & 0 & 3 & 0 & 0 \\
\hline P. value (Wilcoxon) & - & 0.039 & 0.027 & - & 0.006 & 0.003 \\
\hline P. value (Friedman) & 0.001 & & & 0.001 & & \\
\hline P. value Mann. Whiteny (before) & 0.19 & & & & & \\
\hline
\end{tabular}




\begin{tabular}{|c|c|c|c|c|c|c|}
\hline & \multicolumn{3}{|l|}{ Intra-oral } & \multicolumn{3}{|l|}{ Extra-oral } \\
\hline & $\begin{array}{l}\text { Session Before } \\
\text { irradiation }\end{array}$ & Fourth session & Eighth Session & $\begin{array}{l}\text { Session Before } \\
\text { irradiation }\end{array}$ & Fourth session & Eighth Session \\
\hline P. value Mann. Whiteny (after) & $\begin{array}{l}0.74 \\
\mathrm{sad}\end{array}$ & & & & & \\
\hline no problem & 15.2 & 18.2 & 33.3 & 27.3 & 30.3 & 33.3 \\
\hline very mild problem & 9.1 & 21.2 & 9.1 & 6.1 & 9.1 & 6.1 \\
\hline mild problem & 18.2 & 6.1 & 6.1 & 9.1 & 6.1 & 9.1 \\
\hline moderate problem & 6.1 & 3 & 0 & 6.1 & 3 & 0 \\
\hline severe problem & 0 & 0 & 3 & 0 & 0 & 0 \\
\hline problem as bad as it can be & 3 & 3 & 0 & 0 & 0 & 0 \\
\hline P. value (Wilcoxon) & - & 0.008 & 0.004 & - & 0.046 & 0.034 \\
\hline P. value (Friedman) & 0.001 & & & 0.001 & & \\
\hline P. value Mann. Whiteny (before) & 0.15 & & & & & \\
\hline \multirow[t]{2}{*}{ P. value Mann. Whiteny (after) } & 0.81 & & & & & \\
\hline & Sense of taste/sn & & & & & \\
\hline no problem & 18.2 & 21.2 & 45.5 & 12.1 & 18.2 & 33.3 \\
\hline very mild problem & 3 & 18.2 & 6.1 & 6.1 & 12.1 & 15.2 \\
\hline mild problem & 12.1 & 3 & 0 & 0 & 12.1 & 0 \\
\hline moderate problem & 9.1 & 6.1 & 0 & 18.2 & 3 & 0 \\
\hline severe problem & 3 & 3 & 0 & 12.1 & 0 & 0 \\
\hline problem as bad as it can be & 6.1 & 0 & 0 & 0 & 3 & 0 \\
\hline P. value (Wilcoxon) & - & 0.006 & 0.005 & - & 0.052 & 0.002 \\
\hline P. value (Friedman) & 0.001 & & & 0.001 & & \\
\hline P. value Mann. Whiteny (before) & 0.41 & & & & & \\
\hline P. value Mann. Whiteny (after) & $\begin{array}{l}0.17 \\
\text { sneezing }\end{array}$ & & & & & \\
\hline no problem & 24.2 & 27.3 & 45.5 & 9.1 & 12.1 & 27.3 \\
\hline very mild problem & 3 & 6.1 & 3 & 12.1 & 21.2 & 15.2 \\
\hline mild problem & 3 & 15.2 & 3 & 9.1 & 9.1 & 0 \\
\hline moderate problem & 6.1 & 3 & 0 & 12.1 & 3 & 6.1 \\
\hline severe problem & 9.1 & 0 & 0 & 3 & 3 & 0 \\
\hline problem as bad as it can be & 6.1 & 0 & 0 & 3 & 0 & 0 \\
\hline P. value (Wilcoxon) & - & 0.01 & 0.029 & - & 0.056 & 0.004 \\
\hline P. value (Friedman) & 0.001 & & & 0.001 & & \\
\hline P. value Mann. Whiteny (before) & 0.58 & & & & & \\
\hline P. value Mann. Whiteny (after) & $\begin{array}{l}0.047 \\
\text { cough }\end{array}$ & & & & & \\
\hline no problem & 21.2 & 30.3 & 42.4 & 24.2 & 33.3 & 36.4 \\
\hline very mild problem & 3 & 9.1 & 6.1 & 6.1 & 3 & 9.1 \\
\hline mild problem & 6.1 & 9.1 & 3 & 9.1 & 6.1 & 3 \\
\hline moderate problem & 9.1 & 3 & 0 & 6.1 & 6.1 & 0 \\
\hline severe problem & 9.1 & 0 & 0 & 3 & 0 & 0 \\
\hline problem as bad as it can be & 3 & 0 & 0 & 0 & 0 & 0 \\
\hline P. value (Wilcoxon) & - & 0.007 & 0.007 & - & 0.02 & 0.016 \\
\hline P. value (Friedman) & 0.001 & & & 0.001 & & \\
\hline P. value Mann. Whiteny (before) & 2 & & & & & \\
\hline \multirow[t]{2}{*}{ P. value Mann. Whiteny (after) } & 0.53 & & & & & \\
\hline & Thick nasal disc & & & & & \\
\hline no problem & 6.1 & 9.1 & 39.4 & 3 & 3 & 33.3 \\
\hline very mild problem & 0 & 9.1 & 12.1 & 0 & 12.1 & 9.1 \\
\hline mild problem & 3 & 15.2 & 0 & 3 & 24.2 & 3 \\
\hline moderate problem & 6.1 & 12.1 & 0 & 30.3 & 9.1 & 3 \\
\hline severe problem & 24.2 & 6.1 & 0 & 12.1 & 0 & 0 \\
\hline problem as bad as it can be & 12.1 & 0 & 0 & 0 & 0 & 0 \\
\hline P. value (Wilcoxon) & - & 0.001 & 0.001 & - & 0.001 & 0.001 \\
\hline P. value (Friedman) & 0.001 & & & 0.001 & & \\
\hline P. value Mann. Whiteny (before) & $0.03 *$ & & & & & \\
\hline P. value Mann. Whiteny (after) & $\begin{array}{l}0.5 \\
\text { dizziness }\end{array}$ & & & & & \\
\hline no problem & 12.1 & 27.3 & 39.4 & 9.1 & 18.2 & 33.3 \\
\hline very mild problem & 6.1 & 9.1 & 12.1 & 9.1 & 15.2 & 9.1 \\
\hline mild problem & 9.1 & 12.1 & 0 & 12.1 & 9.1 & 6.1 \\
\hline moderate problem & 15.2 & 3 & 0 & 12.1 & 6.1 & 0 \\
\hline severe problem & 6.1 & 0 & 0 & 6.1 & 0 & 0 \\
\hline problem as bad as it can be & 3 & 0 & 0 & 0 & 0 & 0 \\
\hline P. value (Wilcoxon) & - & 0.001 & 0.001 & - & 0.005 & 0.003 \\
\hline P. value (Friedman) & 0.001 & & & 0.001 & & \\
\hline
\end{tabular}




\begin{tabular}{|c|c|c|c|c|c|c|}
\hline & \multicolumn{3}{|l|}{ Intra-oral } & \multicolumn{3}{|l|}{ Extra-oral } \\
\hline & $\begin{array}{l}\text { Session Before } \\
\text { irradiation }\end{array}$ & Fourth session & Eighth Session & $\begin{array}{l}\text { Session Before } \\
\text { irradiation }\end{array}$ & Fourth session & Eighth Session \\
\hline P. value Mann. Whiteny (before) & \multicolumn{6}{|c|}{0.74} \\
\hline P. value Mann. Whiteny (after) & \multicolumn{6}{|c|}{$\begin{array}{l}0.5 \\
\text { facial pain/pressure }\end{array}$} \\
\hline no problem & 3 & 9.1 & 36.4 & 3 & 3 & 24.2 \\
\hline very mild problem & 0 & 15.2 & 15.2 & 0 & 9.1 & 15.2 \\
\hline mild problem & 3 & 12.1 & 0 & 0 & 12.1 & 9.1 \\
\hline moderate problem & 15.2 & 9.1 & 0 & 12.1 & 18.2 & 0 \\
\hline severe problem & 18.2 & 6.1 & 0 & 21.2 & 3 & 0 \\
\hline problem as bad as it can be & 12.1 & 0 & 0 & 12.1 & 3 & 0 \\
\hline P. value (Wilcoxon) & - & 0.001 & 0.001 & - & 0.001 & 0.001 \\
\hline P. value (Friedman) & \multicolumn{3}{|l|}{0.001} & \multicolumn{3}{|l|}{0.001} \\
\hline P. value Mann. Whiteny (before) & \multicolumn{6}{|l|}{0.63} \\
\hline P. value Mann. Whiteny (after) & $\begin{array}{l}0.14 \\
\text { waking up at nigh }\end{array}$ & \multicolumn{4}{|c|}{ waking up at night } & \\
\hline no problem & 12.1 & 12.1 & 36.4 & 30.3 & 33.3 & 36.4 \\
\hline very mild problem & 12.1 & 30.3 & 12.1 & 6.1 & 12.1 & 12.1 \\
\hline mild problem & 15.2 & 9.1 & 3 & 3 & 3 & 0 \\
\hline moderate problem & 9.1 & 0 & 0 & 6.1 & 0 & 0 \\
\hline severe problem & 3 & 0 & 0 & 3 & 0 & 0 \\
\hline problem as bad as it can be & 0 & 0 & 0 & 0 & 0 & 0 \\
\hline P. value (Wilcoxon) & - & 0.058 & 0.004 & - & 0.063 & 0.05 \\
\hline P. value (Friedman) & \multicolumn{3}{|l|}{0.001} & \multicolumn{3}{|l|}{0.001} \\
\hline P. value Mann. Whiteny (before) & \multicolumn{6}{|l|}{$0.07 *$} \\
\hline P. value Mann. Whiteny (after) & \multicolumn{6}{|l|}{0.71} \\
\hline & \multicolumn{6}{|l|}{ Waking up tired } \\
\hline no problem & 12.1 & 12.1 & 27.3 & 18.2 & 18.2 & 24.2 \\
\hline very mild problem & 3 & 15.2 & 15.2 & 3 & 9.1 & 12.1 \\
\hline mild problem & 9.1 & 9.1 & 6.1 & 0 & 12.1 & 12.1 \\
\hline moderate problem & 9.1 & 9.1 & 3 & 18.2 & 9.1 & 0 \\
\hline severe problem & 12.1 & 6.1 & 0 & 6.1 & 0 & 0 \\
\hline problem as bad as it can be & 6.1 & 0 & 0 & 3 & 0 & 0 \\
\hline P. value (Wilcoxon) & - & 0.024 & 0.002 & - & 0.01 & 0.007 \\
\hline P. value (Friedman) & 0.001 & & & 0.001 & & \\
\hline P. value Mann. Whiteny (before) & 0.44 & & & & & \\
\hline P. value Mann. Whiteny (after) & 0.81 & & & & & \\
\hline & lower performanc & & & & & \\
\hline no problem & 12.1 & 15.2 & 33.3 & 15.2 & 21.2 & 30.3 \\
\hline very mild problem & 9.1 & 21.2 & 9.1 & 9.1 & 9.1 & 6.1 \\
\hline mild problem & 12.1 & 9.1 & 6.1 & 6.1 & 12.1 & 6.1 \\
\hline moderate problem & 12.1 & 3 & 3 & 15.2 & 6.1 & 6.1 \\
\hline severe problem & 3 & 3 & 0 & 3 & 0 & 0 \\
\hline problem as bad as it can be & 3 & 0 & 0 & 0 & 0 & 0 \\
\hline P. value (Wilcoxon) & - & 0.01 & 0.002 & - & 0.024 & 0.042 \\
\hline P. value (Friedman) & 0.001 & & & 0.001 & & \\
\hline P. value Mann. Whiteny (before) & 0.64 & & & & & \\
\hline P. value Mann. Whiteny (after) & 0.76 & & & & & \\
\hline & frustrated/restless & rritable & & & & \\
\hline no problem & 18.2 & 27.3 & 33.3 & 15.2 & 18.2 & 33.3 \\
\hline very mild problem & 6.1 & 3 & 9.1 & 3 & 12.1 & 9.1 \\
\hline mild problem & 9.1 & 9.1 & 6.1 & 12.1 & 12.1 & 3 \\
\hline moderate problem & 9.1 & 9.1 & 3 & 15.2 & 6.1 & 3 \\
\hline severe problem & 3 & 3 & 0 & 3 & 0 & 0 \\
\hline problem as bad as it can be & 6.1 & 0 & 0 & 0 & 0 & 0 \\
\hline P. value (Wilcoxon) & - & 0.009 & 0.003 & - & 0.014 & 0.007 \\
\hline P. value (Friedman) & 0.001 & & & 0.001 & & \\
\hline P. value Mann. Whiteny (before) & 0.97 & & & & & \\
\hline P. value Mann. Whiteny (after) & $\begin{array}{l}0.77 \\
\text { embarrassed }\end{array}$ & & & & & \\
\hline no problem & 6.1 & 15.2 & 27.3 & 27.3 & 27.3 & 30.3 \\
\hline very mild problem & 18.2 & 24.2 & 15.2 & 6.1 & 9.1 & 12.1 \\
\hline mild problem & 21.2 & 9.1 & 6.1 & 9.1 & 9.1 & 6.1 \\
\hline moderate problem & 3 & 0 & 0 & 3 & 3 & 0 \\
\hline severe problem & 0 & 0 & 0 & 3 & 0 & 0 \\
\hline problem as bad as it can be & 3 & 3 & 3 & 0 & 0 & 0 \\
\hline $\begin{array}{l}\text { P. value (Wilcoxon) } \\
\text { P }\end{array}$ & - & 0.024 & 0.004 & - & 0.18 & 0.046 \\
\hline
\end{tabular}




\begin{tabular}{|c|c|c|c|c|c|c|}
\hline & \multicolumn{3}{|l|}{ Intra-oral } & \multicolumn{3}{|l|}{ Extra-oral } \\
\hline & $\begin{array}{l}\text { Session Before } \\
\text { irradiation }\end{array}$ & Fourth session & Eighth Session & $\begin{array}{l}\text { Session Before } \\
\text { irradiation }\end{array}$ & Fourth session & Eighth Session \\
\hline P. value (Friedman) & \multicolumn{3}{|l|}{0.001} & \multicolumn{3}{|l|}{0.001} \\
\hline P. value Mann. Whiteny (before) & \multicolumn{6}{|l|}{0.056} \\
\hline P. value Mann. Whiteny (after) & \multicolumn{6}{|c|}{0.54} \\
\hline & \multicolumn{6}{|c|}{ Blockage/congestion of nose } \\
\hline no problem & 6.1 & 6.1 & 33.3 & 3 & 6.1 & 24.2 \\
\hline very mild problem & 3 & 15.2 & 18.2 & 9.1 & 3 & 15.2 \\
\hline mild problem & 9.1 & 18.2 & 0 & 12.1 & 18.2 & 9.1 \\
\hline moderate problem & 24.2 & 12.1 & 0 & 21.2 & 18.2 & 0 \\
\hline severe problem & 9.1 & 0 & 0 & 3 & 3 & 0 \\
\hline problem as bad as it can be & 0 & 0 & 0 & 0 & 0 & 0 \\
\hline P. value (Wilcoxon) & - & 0.002 & 0.001 & - & 0.028 & 0.001 \\
\hline P. value (Friedman) & \multicolumn{3}{|l|}{0.001} & \multicolumn{3}{|l|}{0.001} \\
\hline P. value Mann. Whiteny (before) & \multicolumn{6}{|l|}{0.33} \\
\hline P. value Mann. Whiteny (after) & \multicolumn{6}{|l|}{0.23} \\
\hline
\end{tabular}

In quantitative evaluation of 22 clinical symptoms in SNOT-22 questionnaires, the average score in intra-oral group was 44 for the first session and 9 for the 8th session. This result in extra-oral radiation was 39 for the first session and 11 for the 8th session. Then Paired T-test was performed on these numbers, and the results showed no significant difference $(\mathrm{P}$. value $=0.41)$. Paired T-test also performed on score of five major symptoms and the results showed significant decrease by intra-oral radiation than extra-oral group $(\mathrm{P}$. value $=0.001)$.

\subsection{The Results of Rhinomanometery}

For evaluating the efficacy of intra and extra-oral laser therapy, we used Wilcoxon Signed Ranks Test to assess the improvement of total air flow and decrease of total air resistance at 150 Pascal pressure. The results obtained in table 2 show that these methods significantly increased total air flow and decreased nasal resistance at inspiration and expiration $(\mathrm{P}$ value $=0.001)$.

Table 2. Results of Wilcoxon test on inhale and exhale at $150 \mathrm{~Pa}$ in extra-oral and intra-oral group.

\begin{tabular}{|c|c|c|c|c|c|}
\hline \multirow{2}{*}{ Rhinomanometry result } & & \multicolumn{2}{|l|}{ inhale } & \multicolumn{2}{|l|}{ exhale } \\
\hline & & first Session & Eight Session & first Session & Eight Session \\
\hline \multirow{6}{*}{$\begin{array}{l}\text { Average total air flow at a } \\
150 \mathrm{~Pa} \\
\text { P. value (Wilcoxon) } \\
\text { Average total resistance at } \\
\text { a } 150 \mathrm{~Pa} \\
\mathrm{P} \text {. value (Wilcoxon) }\end{array}$} & Intra-oral & 86.52 & 285.21 & 141.53 & 458.68 \\
\hline & Extra-oral & 82.38 & 225.58 & 140.60 & 231.03 \\
\hline & & .001 & & .001 & \\
\hline & Intra-oral & 3.3059 & .7862 & 3.0600 & .5024 \\
\hline & Extra-oral & 3.5913 & 1.2350 & 3.0475 & .8874 \\
\hline & & .001 & & .001 & \\
\hline
\end{tabular}

After that, Non-parametric Mann-Whitney test was also used to compare intra and extra-oral irradiation (table 3). Before treatment, the average of total air flow and nasal resistance did not show significant differences. At the end of treatment, intraoral group increased total air flow and decreased nasal resistance significantly.

Table 3. Results of Mann-Whitney test in comparison of extra-oral and intra-oral group.

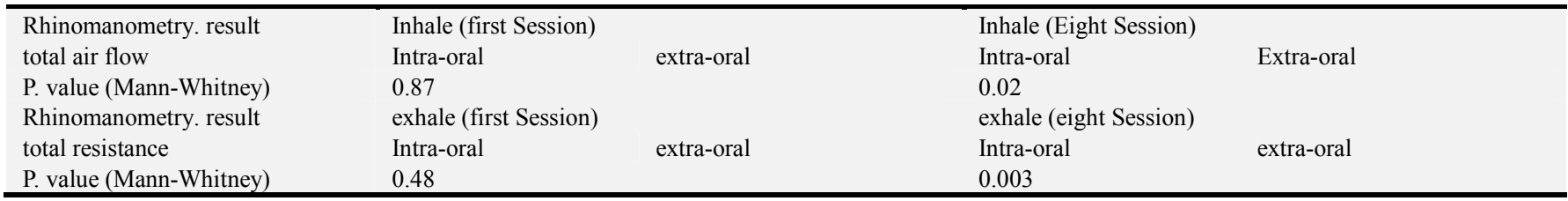

\subsection{The Results of Follow-up}

Evaluating the data obtained six months after treatment by SNOT-22 questionnaires and comprising these data with the results obtained in 8 th session showed that the patients in both groups reported a small reduction in clinical symptoms and there was no significant difference in comparison to 8th session of treatment by Wilcoxon test $(\mathrm{P}$ value $>0.05)$, except two clinical symptoms "post nasal discharge and thick nasal discharge" which decreased significantly in comparison to the 8th session in extra-oral radiation. (P. value $=.02$, pvalue $=.005)$

\section{Discussion}

This study entitled "Comparison of therapeutic effects of intra and extra oral low level laser radiation in maxillary chronic sinusitis" Was carried out to answer the following three questions:

1. Is the use of intra-oral low level lasers in the treatment of chronic maxillary sinusitis effective?

2 . Is the use of extra-oral low level lasers in the treatment 
of chronic maxillary sinusitis effective?

3 . Is there a significant difference between them?

The results of intraoral oral irradiation (alone), due to the importance of the subject and its novelty, have been published in another paper [8], and in this paper, the results of two types of radiation (in-oral-extra-oral) are compared.

Low-level diode laser with $810 \mathrm{~nm}$ wavelength and output power of $0.1,0.2,0.3$ and $0.4 \mathrm{w}$ was used to treat chronic maxillary sinusitis. This treatment repeated for 40 minutes in 8 sessions (60 seconds irradiation in 20 points on both sides plus rest time). So, depending on different output powers, received dose for every patient was between $4-7 \mathrm{~J} / \mathrm{cm}^{2}$. The reason of using this dose is that doses lower than $4 \mathrm{~J} / \mathrm{cm}^{2}$ are not effective on inflamed tissues [8].

Results show that intra-oral radiation significantly decreased all 22 clinical symptoms included in SNOT-22 questionnaires. And details of the improvement of each of these items have been reported in the previous article [8]

Briefly, mean score of SNOT-22 questionnaire for all patients were 44 . This score was in accordance with the score range of patients with chronic sinusitis in other studies. According to the previous studies, mean score of healthy people with the age range of 19-75 was 9.3 and for the age range of $18-24$ it was 8.06 . After 8 treatment sessions in this study, mean score of intra-oral irradiation group was 9 which was in accordance with Gillete study in 2009 [17, 18].

Based on rhinomanometry results, intra-oral irradiation significantly decreased total resistance of nasal cavity and increased total air flow. According to the different studies such as Broms and Suzina, the mean and range of the resistance changes in healthy people were $0.24(-0.52-0.12)$ $[19,20]$ which was not accordant with the results of our study. In the end of 8th treatment session, airway resistance was 0.7 and 0.5 in inspiration and expiration, respectively.

In response to the second question posed in our study, we can say that extra-oral laser irradiation in treatment of chronic maxillary sinusitis was not new a method and previous studies have also used it in two ways: from facial skin Like Naghdi [6] and Isser's [7] studies and intra-nasal radiation that used by Krepsi. [21]. Of course Laser radiation from skin is less invasive and more acceptable by patients.

Results show that this type of irradiation decreased significantly all 22 clinical symptoms same as intra-oral radiation.

Need to blow nose, post nasal discharge, thick nasal discharge, facial pressure/ pain and sense of taste/smell were reduced significantly in second session. These results were consistent with the findings of Krespi [21] and Isser [7].

A significant decrease in sneezing, cough, blockage/congestion of nose and ear fullness was seen in fifth session that these findings are consistent with the results of Naghdi's [6] study and began to decrease significantly more slowly compared with Krepsi's [21] finding.

In addition to the above variables, runny nose and ear pain in this group were decreased significantly in the third session and these findings are consistent with result of Krepsi [21].

Sleep related variables (Lack of a good night's sleep,
Difficulty falling asleep, Waking up at night, Waking up tired) decreased significantly in the third, seventh and second, respectively. But this type of irradiation decreased severe and very severe form of sleep related variables to zero in the third, second, and seventh sessions. So, these results are consistent with the findings of Krepsi [21] except waking up at night.

But in Naghdi's [6] study Overall discomfort variable which includes sleep disorders decreased significantly in the 10th session. To justify this difference, it can be said that long exposure time in this study can be the reason.

As mentioned earlier, quality of life is affected by chronic sinusitis [22, 23]. So psychological factors were also considered in evaluating the efficiency of different therapeutic modality in sinusitis. In patients with extra-oral irradiation, fatigue, reduced concentration, frustration/restlessness decreased significantly in the second session and low performance decreased significantly in the third session. Sadness and embarrassment were significantly reduced in the fourth and seventh session respectively. Therefore, this type of treatment also decreased psychological symptoms like disease related symptoms.

Before extra oral laser therapy, mean score of SNOT-22 questionnaire for all patients in this group was 39 that decreased to 11 by completion of treatment which was higher than the score of healthy individuals in past available studies. $[17,18]$.

Extra-oral irradiation significantly decreased resistance of nasal cavity and increased total air flow. At the end of 8th session, airway resistance was 1.2 and 0.9 in inspiration and expiration respectively which was not accordant with the results of other studies. $[17,18]$ the increase of total air flow was consistent with the results of previous studies. [24].

By comparing intra and extra-oral radiation, in response to the third question, it is concluded that intraoral laser radiation increased total air flow and reduced total nasal resistance significantly, so in comparison with extra-oral, it has greater therapeutic effect. (Table 3).

In previous studies such as wollfwork in 2006 [25], similar tests to rhinomanometry were used to evaluate efficacy of macrolides in treatment of chronic sinusitis. The results showed that, despite efficiency of macrolides in treatment of this disease, air flow had not increased significantly, so this finding was not consistent with our study.

In surveying SNOT-22 variables, these two methods differ only in the sneezing variable which significantly decreased by intra-oral radiation. ( $\mathrm{P}$ value $=0.047$ ).

All major clinical symptoms reduced significantly in the second session in intra and extra group, except nasal obstruction that decreased significantly in the fifth session in extra-oral group.

Another point that needs to be pointed is the average mean of all the variables, except fatigue, reduced concentration, frustration/restlessness and sense of taste/smell that before exposure to laser radiation was higher in intra-oral group than extra-oral group and this ratio is reversed at the end of 8 th session. 
Clinical efficacy of intra-oral low-level laser irradiation, which was the most important hypothesis of this study, was suggested and proved. Maxilla's vestibule depth is in fact the floor of maxillary sinus and sinus discharges collected in this zone because of gravity. Thus, using suitable irradiation degree, this zone benefited much from anti-inflammatory effects of laser. On the other hand, thickness and color of skin, the thickness of underlying muscles and interactions of zygomatic arch which are important in extra-oral irradiation in other studies $[6,7,21,26]$ do not affect the intra-oral laser therapy.

The results of the 8th session did not differ with the result of follow-up duration in intra-oral group, but in extra-oral group thick nasal discharge and post nasal discharge decreased compare with the 8th session. However, it does not mean that the problem is same as pre-radiation time, but it means this variable only reduced in comparison to the 8th session's result.

Among 12 studies in this regard, all studies except Moustsen [27] reported positive therapeutic effects of lowlevel laser therapy in the treatment of acute and chronic sinusitis. The results of our study were also in accordance with previous studies.

In the following, laser parameters such as wavelength, energy density, power and the duration of irradiation for each patient should be chosen individually. Lack of appropriate selection of these parameters reduce the effectiveness of laser therapy.

In this study, Diode laser with $810 \mathrm{~nm}$, output power of $(0.1,0.2,0.3$, and 0.4$) \mathrm{w}$ for 20 minutes was used in the treatment of chronic sinusitis maxillary.

The radiation dose which received by every patient (output power) ranged from $4-7 \mathrm{~J} / \mathrm{cm}^{2}$ since the energy density of less than $4 \mathrm{~J} / \mathrm{cm}^{2}$ does not have favorable effects on inflamed tissues. [8]

In all previous studies, Helium-neon and Diode laser with 830 and $810 \mathrm{~nm}$ were used. So our study was similar to Isser's study [7], and differ from Krepsi [21], Nghdi [6] and Hacarova studies [28].

The exposure time and number of sessions in our study were very longer than previous studies, so it could not be compared with them.

Finally the results of rhinomanometry with the results of SNOT-22 questionnaire had no significant relationship. This finding was consistent with the findings of several studies in which lack of a significant relationship between the results of clinical assessment and objective findings as the results of $\mathrm{CT}$, endoscopy and rhinomanometry, had been achieved.

\section{Conclusion}

Treatment with intra-oral and extra-oral radiation causes a statistically significant and obvious improvement of clinical symptoms as well as a significant increase in total air flow and a significant reduction in nasal resistance in patients affected by chronic maxillary sinusitis and between these two types of radiation, Intraoral radiation had more clinical efficacy. Using low-level laser in treatment of chronic maxillary sinusitis is a suitable and conservative choice and also has no side effects. Stability of treatment is desirable according to the results of follow up.

\section{References}

[1] Kim W Ah-See, Andrew S Evans. Sinusitis and its management. British Medical Journal. 2007 Feb 17; 334 (7589): 358-361.

[2] Savastano V, Bertin S, Vittori T, Tripodi C, Magliulo G. Evaluation of chronic rhinosinusitis management using the SNOT-22 in adult cystic fibrosis patients. Eur Rev Med Pharmacol Sci. 2014; 18 (14): 1985-9.

[3] Farhood Z, Schlosser RJ, Pearse ME, Storck KA, Nguyen SA, Soler ZM Twenty-two-item Sino-Nasal Outcome Test in a control population: a cross-sectional study and systematic review. Int Forum Allergy Rhinol. 2016 Mar; 6 (3): 271-7.

[4] Cain RB, Lal D. Update on the management of chronic rhinosinusitis. Infect Drug Resist. 2013; 6: 1-14.

[5] John Malaty. Medical Management of Chronic Rhinosinusitis in Adult. Sinusitis 2016, 1 (1), 76-87.

[6] Naghdi S, Ansari NN, Fathali M, Bartley J, Varedi M, Honarpishe R. A pilot study into the effect of low-level laser therapy in patients with chronic rhinosinusitis. Physiother Theory Pract. 2013 Nov; 29 (8): 596-603.

[7] Isser DK, Sett S, Saha BP. The role of laser radiation therapy in maxillary sinusitis: Indian J Otolaryngol Head Neck Surg. 2002; 54: 208-15.

[8] Mortazavi H, Khalighi H, Goljanian A, Noormohammadi R, Mojahedi S, Sabour s. Intra-oral low level laser therapy in chronic maxillary sinusitis: A new and effective recommended technique. J Clin Exp Dent. 2015 Dec 1; 7 (5): e557-62.

[9] Howard B Cotler, Roberta T Chow, Michael R Hamblin, James Carroll. The Use of Low Level Laser Therapy (LLLT) For Musculoskeletal Pain. MOJ Orthop Rheumatol. 2015; 2 (5): 00068.

[10] Krespi Y, Kizhner V. Phototherapy for chronic rhinosinusitis. Lasers Surg Med. 2011; 43: 187-91.

[11] Benninger MS, Ferguson BJ, Hadley JA, Hamilos DL, Jacobs M, Kennedy DW, et al. Adult chronic rhinosinusitis: definitions, diagnosis, epidemiology, and pathophysiology. Otolaryngol Head Neck Surg. 2003; 129: S1-32.

[12] Rathora A, Bhattacharjee A. Clinical-radiological correlation and role of computed tomography staging in chronic rhinosinusitis. World J Otorhinolaryngol Head Neck Surg. 2017 Sep; 3 (3): 169-175.

[13] Zheng Y, Zhao Y, Lv D, Liu Y, Qiao X, An P, et al. Correlation between computed tomography staging and quality of life instrumentsin patients with chronic rhinosinusitis. Am J Rhinol Allergy. 2010; 24: 41-5.

[14] Suh JD, Kennedy DW. Treatment Options for Chronic Rhinosinusitis. Proc Am Thorac Soc. 2011; 8: 132-40.

[15] Hopkins C, Browne JP, Slack R, Lund V, Topham J, Reeves B, et al. The national comparative audit of surgery for nasal polyposis and chronic rhinosinusitis. Clin Otolaryngol. 2006. 31: 390-8. 
[16] Hopkins C, Gillett S, Slack R, Lund VJ, Browne JP. Psychometric validity of 22-item sinonasal outcome test. Clin Otolaryngol. 2009; 34: 447-54.

[17] Proimos E, Papadakis CE, Chimona TS, Kiagiadaki D, Ferekidis E, Yiotakis J. The effect of functional endoscopic sinus surgery on patients with asthma and CRS with nasal polyps. Rhinology. 2010; 48: 331-8.

[18] Gillett S, Hopkins C, Slack R, Browne JP. A pilot study of the SNOT 22 score in adults with no sinonasal disease. Clin Otolaryngol. 2009; 34: 467-9.

[19] Suzina AH, Hamzah M, Samsudin AR. Objective assessment of nasal resistance in patients with nasal disease. J Laryngol Otol. 2003; 117: 609-13.

[20] Broms P, Jonson B, Malm L. Rhinomanometry. IV. A pre- and postoperative evaluation in functional septoplasty. Acta Otolaryngol. 1982, 94: 523-9.

[21] Krespi Y, Kizhner V. Phototherapy for chronic rhinosinusitis. Lasers in Surgery and Medicine 2011.43 (3): 187-191.

[22] Benninger MS, Ferguson BJ, Hadley JA, Hamilos DL, Jacobs M, Kennedy DW, et al. Adult chronic rhinosinusitis: definitions, diagnosis, epidemiology, and pathophysiology. Otolaryngol Head Neck Surg. 2003 129: S1-32.
[23] Caminha GP, Melo Junior JT, Hopkins C, Pizzichini E, Pizzichini M. SNOT- 22: psychometric properties and crosscultural adaptation into the protugues language spoken in Brazil. Braz J Otorhinolaryngol. 2012; 78: 34-9.

[24] Eccles R. Nasal airflow in health and disease. Acta Otolaryngol. $2000,120: 580-95$.

[25] Wallwork B, Coman W, Mackay-Sim A, Greiff A, CervinA. A Double-Blind, Randomized, Placebo-Controlled Trial of Macrolide in the Treatment of Chronic Rhinosinusitis. Laryngoscope; 2006, 3: 116.

[26] Pekli FF, Kruchinina IL. Physiological functions of the nose before and after laser treatment of acute and chronic maxillary sinusitis in children. Vestn Otorinolaringol. 1988, (3): 53-5.

[27] Moustsen PA, Vinter N, Aas-Andersen L, Kragstrup J. Laser Treatment of Sinusitis in General Practice Assessed by a Double-blind Controlled Study. Ugeskr Laeger. 1991; 153: 2232-4.

[28] Hacarova. Low Level Laser Therapy of Sinusitis. 2002. Dobnerova 26, Olomouc, CZ. J. Hubacek, Faculty Hospital, ENT Clinic, I. P. Pavlova 6, Olomouc, CZ. 